\title{
Troponin I Levels in Extracorporeal Membrane Oxygenation Following Congenital Heart Surgery
}

World Journal for Pediatric and

Congenital Heart Surgery

2014, Vol. 5(2) 229-235

(C) The Author(s) 2013

Reprints and permission:

sagepub.com/journalsPermissions.nav DOI: $10.1177 / 2150135113510007$ pch.sagepub.com

(SAGE

\author{
André Rüffer, MD', Frank Münch', Sergej Potapov, MSc $^{2}$, \\ Ariawan Purbojo, MD', Okan Toka, MD ${ }^{3}$, \\ Ali Dodge-Khatami, MD, $\mathrm{PhD}^{4}$, Sven Dittrich, MD, $\mathrm{PhD}^{3}$, \\ and Robert Anton Cesnjevar, MD, PhD'
}

\begin{abstract}
Background: Correlating postcardiotomy extracorporeal membrane oxygenation (ECMO) troponin I (Tnl) levels and outcomes. Methods: Between January 2006 and August 2010, 34 patients needed postcardiotomy ECMO for low cardiac output. Bailout ECMO was required either after unsuccessful weaning from bypass $(n=17,50 \%)$, postoperatively from prolonged hemodynamic failure $(n=8,23.5 \%)$, or following resuscitation $(n=9,26.5 \%)$. The Tnl levels were measured following surgery or resuscitation during 10 days and compared between survivors (group I) and non-survivors (group II). Results: Median support duration was seven days (range: $0-3 \mathrm{I}$ ). Surgery involving hypoplastic aortic arch repair (Norwood palliation; $\mathrm{n}=7,20.6 \%$, or biventricular repair; $\mathrm{n}=1 \mathrm{I}, 32.4 \%$ ) led to most ECMO runs. Successful weaning from ECMO and hospital survival were $76.5 \%$ and $50 \%$, respectively. In group I, peak Tnl levels were reached by 24 hours postoperatively, comparable to levels in group II $(36 \pm 34$ vs $49 \pm 38 \mathrm{ng} / \mathrm{mL} ; P=.98)$. However, in group II, Tnl levels formed a plateau by the second postoperative day, whereas group I showed a steep decline in Tnl levels, suggesting myocardial recovery $(P=.028)$. All patients $(n=4)$ who reached or maintained peak Tnl levels at 48 hours died. On days 8,9 and I0, Tnl levels were significantly higher in group II $(P=.024, .019$, and .013 , respectively). Conclusions: Postcardiotomy ECMO was most commonly required after aortic arch repair. In the absence of ongoing myocardial insult due to ECMO hardware issues, coronary insufficiency or residual lesions, plateau Tnl levels at 48 hours may seem to indicate an unfavorable outcome due to irreversible myocardial damage.
\end{abstract}

\section{Keywords}

extracorporeal membrane oxygenation, troponin, congenital heart surgery, aortic arch

Submitted June 3, 20I3; Accepted September 30, 2013.

\section{Introduction}

Extracorporeal membrane oxygenation (ECMO) has been widely used for resuscitation after repair of congenital heart defects. ${ }^{1}$ Despite huge advances in ECMO hardware technology, intensive care unit (ICU) management, and more judicious indications to deploy ECMO, weaning from cardiac ECMO after congenital heart surgery or after rapid resuscitation for acute cardiac failure of any origin still ranges only between $56 \%$ and $79 \%{ }^{2-6}$ Survival to hospital discharge is unfortunately even lower, ranging between $38 \%$ and $59 \% .^{2-7}$ While increasingly precise protocols are being developed to guide ECMO management, it is still difficult to predict, in the first days after ECMO implantation, whether cardiac recovery is likely or not. Reliable predictors for successful weaning from extracorporeal life support are rare and need to be defined.
Troponin I (TnI) is a specific marker of cardiac myocyte damage and has no detectable cross-reactivity with the skeletal

\footnotetext{
'Department of Pediatric Cardiac Surgery, University Hospital Erlangen, Erlangen, Germany

${ }^{2}$ Department of Medical Informatics, Biometry and Epidemiology, FriedrichAlexander-University of Erlangen-Nuremberg, Erlangen, Germany

${ }^{3}$ Department of Pediatric Cardiology, University Hospital Erlangen, Erlangen, Germany

${ }^{4}$ Division of Pediatric and Congenital Heart Surgery, Batson Children's Hospital, University of Mississippi Medical Center, Jackson, MS, USA
}

\section{Corresponding Author:}

André Rüffer, Department of Pediatric Cardiac Surgery, University Hospital Erlangen, Loschgestrasse 5I, 91054 Erlangen, Germany.

Email: andre.rueffer@uk-erlangen.de 


\section{Acronyms and Abbreviations \\ ASD atrial septal defect \\ ECMO extracorporeal membrane oxygenation \\ ELSO Extracorporeal Life Support Organization \\ ICU intensive care unit \\ Tnl troponin I}

muscle isoforms. ${ }^{8,9}$ Elevated blood levels are associated with adverse outcome in patients with heart failure and cardiogenic shock complicating myocardial infarction, ${ }^{10}$ and predict mortality in coronary artery bypass surgery. $8,11,12$ Particularly in children, absolute values of TnI correlate very well with the extent of perioperative myocardial damage. ${ }^{13,14}$

This study was performed to determine whether the postoperative course of TnI in children on postcardiotomy ECMO therapy could serve as a biochemical marker to reflect reversibility of perioperative myocardial damage and thus provide a prognostic indicator for cardiac recovery and successful weaning from ECMO.

\section{Materials and Methods}

\section{Patients}

The institutional review board of our hospital approved retrospective anonymous chart analysis for this study, and parental consent was waived. Accordingly, the records of 34 pediatric patients who required postcardiotomy ECMO therapy due to low cardiac output between January 2006 and August 2010 at the University Hospital Erlangen, Germany, were retrospectively reviewed. Median age and weight were 25.5 days (range 4-921 days) and $3.7 \mathrm{~kg}$ (range 2.1-12.0 kg), respectively. The majority of patients were neonates $(\mathrm{n}=18,52.9 \%)$ or infants ( $\mathrm{n}=13,38.2 \%$ ), and only three children (8.8\%) were older than one year $(1.7,1.9$, and 2.5 years old). Bailout ECMO implantation occurred either intraoperatively after unsuccessful weaning from bypass $(\mathrm{n}=17,50 \%)$ or postoperatively as a result of prolonged low cardiac output $(n=8,23.5 \%)$ or following rapid resuscitation $(\mathrm{n}=9,26.5 \%)$.

\section{Extracorporeal Membrane Oxygenation}

Mechanical cardiopulmonary support was established by venoarterial ECMO, which was deployed via median sternotomy and direct aortic and right atrial cannulation. The ECMO flow was adjusted to patient's body surface area $\left(\mathrm{L} / \mathrm{m}^{2} / \mathrm{min}\right)$ multiplied by factor $3.0\left(\mathrm{~L} / \mathrm{m}^{2} / \mathrm{min}\right)$ for patients $<4 \mathrm{~kg}$, factor $2.8\left(\mathrm{~L} / \mathrm{m}^{2} / \mathrm{min}\right)$ for patients between 4 and $10 \mathrm{~kg}$, and factor $2.6\left(\mathrm{~L} / \mathrm{m}^{2} / \mathrm{min}\right)$ for patients $>10 \mathrm{~kg}$. Full-flow ECMO was maintained until cardiac recovery was suggested. Indicators to predict successful weaning from extracorporeal hemodynamic support included daily transthoracic or transesophageal echocardiography for assessment of global and regional cardiac function, an increasing area under the curve using invasive measurement of the arterial blood pressure as a marker of systemic ventricular recovery, increasing end-expiratory $\mathrm{CO}_{2}$ marking improvement in right heart function with improved pulmonary blood flow. Contrarily, elevated or increasing lactate levels or decreasing mixed venous saturations during weaning pointed to inadequate systemic perfusion resulting from insufficient cardiac function. Additionally, the course of TnI was followed, and elevated levels were considered as surrogates of persisting cardiac ischemia, suggesting failure to recover. Progressive weaning from ECMO was attempted during a three-day interval. Minimal flow was maintained for 24 hours depending on the body surface area and calculated oxygenator sizes, at 200,300 , or $1,000 \mathrm{~mL} / \mathrm{min}$, accordingly.

\section{Troponin I}

The TnI was measured in all children undergoing surgery for congenital heart defects when ECMO therapy was necessary for intra- or postoperative hemodynamic support. The course of TnI levels was observed immediately after ECMO implantation and daily until ICU discharge and was compared between hospital survivors (group I) and nonsurvivors (group II). Additionally, in order to represent the perioperative course of TnI levels after routine successful pediatric cardiac surgery, a control group of all hospital survivors operated in 2010 at an age younger than one year without the need of ECMO and in whom TnI levels were available was added (group III).

Laboratory values were measured by the clinical laboratory of the University of Erlangen-Nuremberg, Germany.

\section{Statistics}

Patients who were alive within 24 hours beyond ECMO decannulation were counted as successfully weaned patients. Early or hospital mortality reflect all deaths within 30 days after ECMO implantation or during hospital stay, respectively. Descriptive data for continuous variables are presented as the mean ( \pm standard deviation) or median (range), and categorical variables are presented as numbers or proportion (\%) of cases. Looking at TnI levels, the "last observation carried forward" method was applied to complete missing levels of TnI. These variables were compared daily between groups I and II. Additionally, in order to compare the individual behavior of TnI levels between groups, the difference in daily decline in levels was calculated. Statistical significance was analyzed using Fisher exact test or chi-square $\left(\chi^{2}\right)$ test for categorical variables and $T$ test or Wilcoxon rank-sum test for continuous variables. Survival analysis was calculated according to the Kaplan-Meier method. All statistical analyses were conducted using the software $\mathrm{R}$ version 2.12.2 ( $\mathrm{R}$ Foundation for Statistical Computing, Vienna, Austria). Statistical significance was defined at a $P$ value of $<.05$.

\section{Results}

\section{Patient Characteristics}

The majority of patients had biventricular morphology $(n=22$, $64.7 \%$ ). The most common cardiac procedure associated with ECMO was biventricular correction of a hypoplastic 


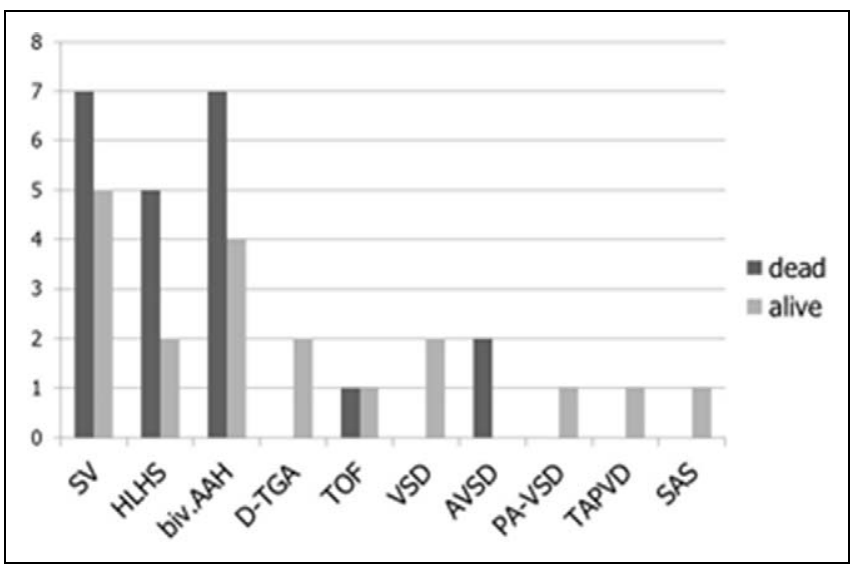

Figure I. Leading cardiac diagnoses. There were no significant differences between groups. AVSD, atrioventricular septal defect; biv aortic arch, biventricular patients with aortic arch hypoplasia; D-TGA, Dtransposition of great arteries; HLHS, hypoplastic left heart syndrome (part of single ventricle); PA-VSD, pulmonary atresia with ventricular septal defect; SAS, supravalvar aortic stenosis; SV, single-ventricle morphology; TAPVD, total anomalous pulmonary venous drainage; TOF, tetralogy of Fallot; VSD, ventricular septal defect.

aortic arch in association with other congenital anomalies $(\mathrm{n}=11,32.4 \%)$ followed by the Norwood procedure $(\mathrm{n}=7$, $20.6 \%$ ). Therefore, $52.9 \%$ of palliated or repaired congenital malformations requiring postcardiotomy ECMO support were related to surgery for congenital aortic arch hypoplasia.

\section{Outcomes}

Weaning from ECMO was successful in $26(76.5 \%)$ patients. In general, patients who could not be weaned from ECMO had persisting global low cardiac function on echocardiography, whereas those who were weaned showed improving contractility. No regional wall motion abnormalities indicating selective coronary injury were diagnosed. Therefore, cardiac catheterization for coronary artery imaging was not performed. In the survival group, low cardiac output seemed to be a sign of myocardial stunning with generalized wall motion abnormalities, which mostly improved during ECMO support as diagnosed by echocardiography.

The 30-day survival was $68.6 \%(n=23)$, and hospital survival $50 \%(\mathrm{n}=17$; group I). There were no significant differences regarding patient characteristics and procedures performed between groups (Figure 1; Table 1), except more aortic arch surgery in the nonsurvivor group (group I: $\mathrm{n}=6$, $35.3 \%$ vs group II: $\mathrm{n}=12,70.6 \% ; P=.034$ ).

In all patients, including those in group II, all technical and mechanical issues potentially pointing to inadequate ECMO performance and myocardial unloading were checked and excluded according to our knowledge.

\section{Further Procedures}

Five patients underwent additional surgery on ECMO, and two had diagnostic cardiac catheterization - none of them survived: two patients had unbalanced atrioventricular canal, one with primary aortopulmonary shunt who received secondary atrioventricular valve reconstruction after four days, and one after failed corrective surgery and secondary right atrioventricular valve reconstruction who was palliated with a takedown and pulmonary artery banding after six days; one patient with aortic arch hypoplasia and ventricular septal defect, who underwent aortic arch patch augmentation and pulmonary artery banding, needed primary ECMO after failed weaning from bypass and was switched to a secondary Norwood procedure due to persisting left heart insufficiency after seven days of support; one patient with transposition of great arteries, ventricular septal defect, and aortic arch hypoplasia, who underwent corrective surgery with an intentional pop-off atrial septal defect (ASD) for left ventricular decompression, required ECMO for persisting low cardiac output on the second postoperative day and underwent secondary ASD closure after two days of support due to increased left-to-right shunting and inadequate left ventricular filling. These four patients had echocardiography while on ECMO as the only diagnostic tool prior to secondary surgical intervention. The last patient with pulmonary atresia and intact ventricular septum was palliated with an aortopulmonary shunt and resuscitated postoperatively on the ICU. During ECMO implantation, acute shunt thrombosis was suspected and verified by subsequent cardiac catheterization; and therefore, shunt revision was performed. Additionally, one patient on ECMO after an arterial switch operation with aortic arch repair underwent diagnostic cardiac catheterization due to elevated TnI levels where coronary artery pathology was excluded.

\section{Troponin I Levels}

Among the survivors in group I, peak TnI levels were reached by 24 hours after surgery and were not significantly different to levels in the nonsurvival group II ( $36 \pm 34$ vs $49 \pm 38 \mathrm{ng} / \mathrm{mL}$, respectively; $P=.98)$. However, on days 8,9 , and 10 , TnI levels were significantly higher in group II $(P=.024, .019$, and .013 , respectively). Additionally in group II, TnI levels formed a plateau between the first and second postoperative day, whereas group I showed a steep decline in TnI levels as a sign of adequate ECMO support and myocardial recovery $(P=.03$; Figures 2 and 3. All patients $(n=4)$ who maintained their peak TnI levels at 48 hours after the operation died (Figure 4).

The control group of 42 patients from 2010 in whom TnI levels were available shows a peak after arrival on ICU and a steep decline in levels until the first postoperative day $(P<.01$; Figure 5).

\section{Comment}

\section{Patient Characteristics}

In this study, $52.9 \%(\mathrm{n}=18)$ of pediatric postcardiotomy EMCOs were used for neonates, followed by $13(38.2 \%)$ infants, and the remaining 3 patients were between 1 and 2 years old. The predominant procedure in our series was biventricular repair in the context of aortic arch surgery and 
Table I. Perioperative Data.

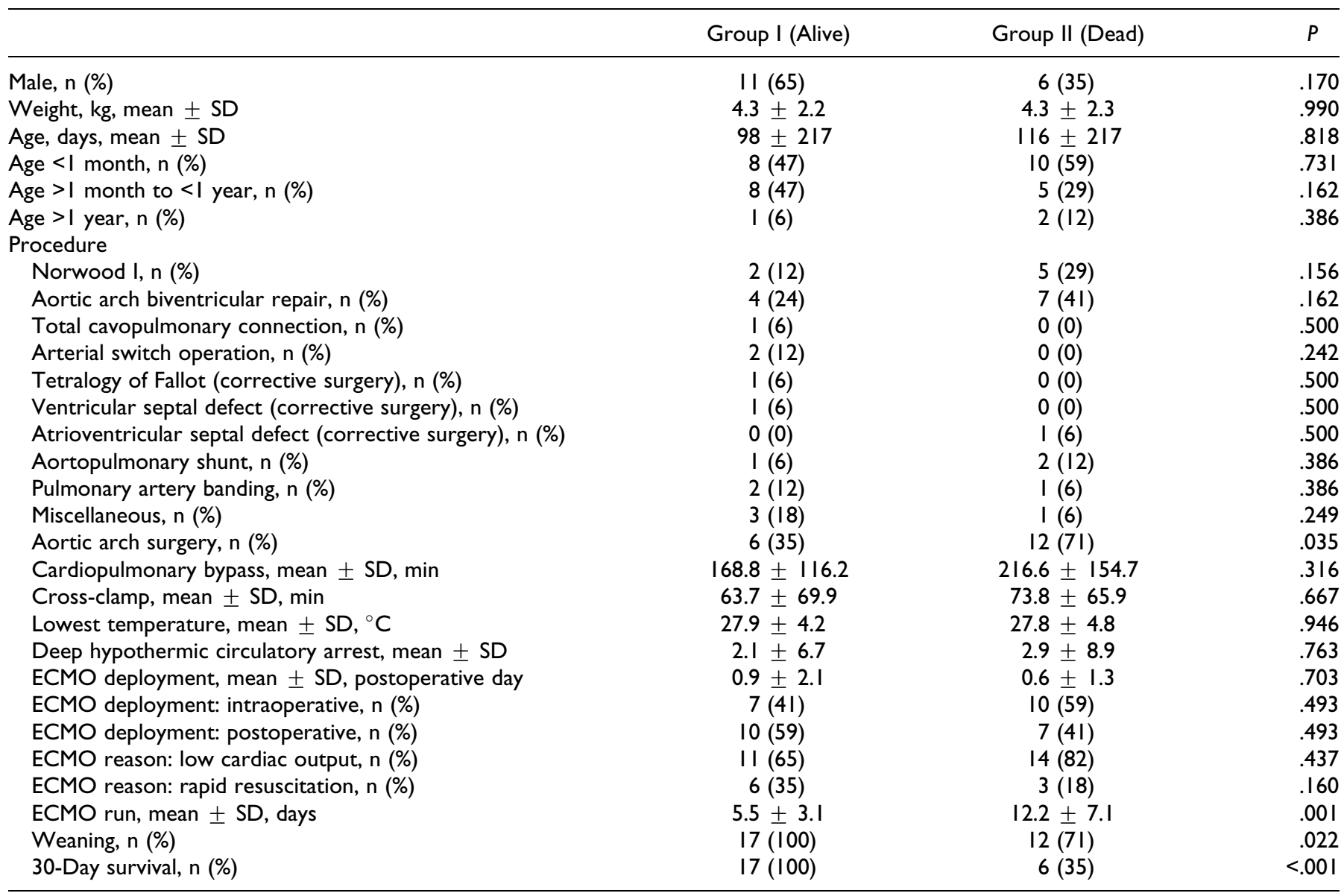

Abbreviations: ECMO, extracorporeal membrane oxygenation; SD, standard deviation.

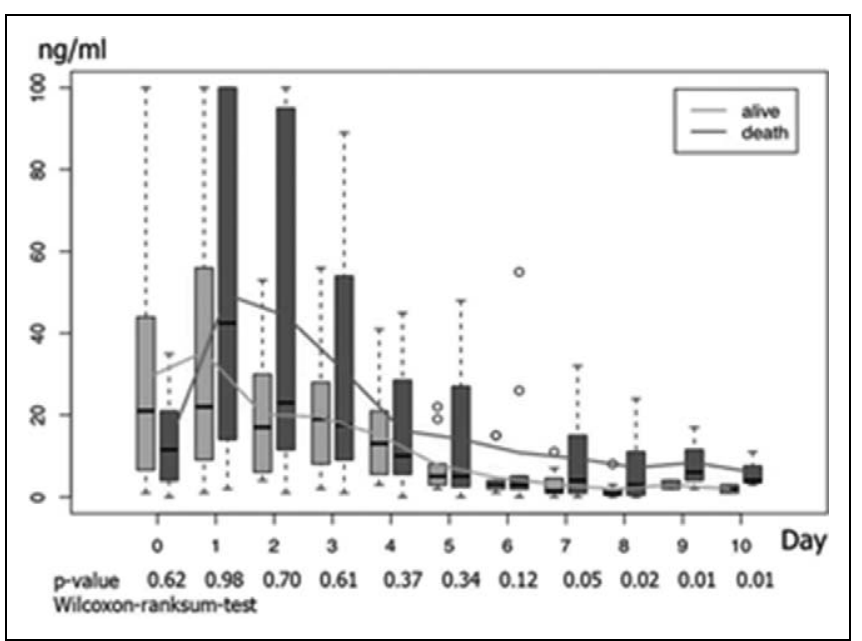

Figure 2. Box plots of perioperative median troponin I levels in extracorporeal membrane oxygenation (ECMO) survivors (light gray) and nonsurvivors (dark gray). $P$ values between groups were counted by Wilcoxon rank sum test for each day.

correction of various intracardiac anomalies (32.4\%), followed by the Norwood palliation (20.6\%). Considered together, repair of hypoplastic aortic arch led to more than half of the ECMO runs. Our patients correspond well to the Extracorporeal Life Support Organization (ELSO) ${ }^{15}$ Registry Report 2008, which included 3,416 neonates as its major age group requiring ECMO support, and revealing the Norwood palliation as its most common procedure. It is unclear from the ELSO registry whether the other most common procedures including the arterial switch had an associated hypoplastic aortic arch.

\section{Outcome}

The percentage of patients successfully weaned from ECMO and surviving at least 24 hours beyond decannulation (76.5\%), until 30 days after ECMO implantation (67.6\%) or to hospital discharge (50\%), is comparable with other studies. ${ }^{2-7}$ Previous reports have defined single-ventricle morphology, ${ }^{2,3,5,16,17}$ younger age, and ECMO deployed for rapid resuscitation, ${ }^{2,4,18}$ as markers for poor outcome. In our study, we could not establish any influence of these parameters on outcome by univariate analysis. Interestingly, aortic arch surgery was more often performed in the nonsurvivor group. We believe that aortic arch hypoplasia is a "symptom" of left ventricular hypoplasia requiring treatment with either a Norwood procedure or by extensive surgery for biventricular repair, and 


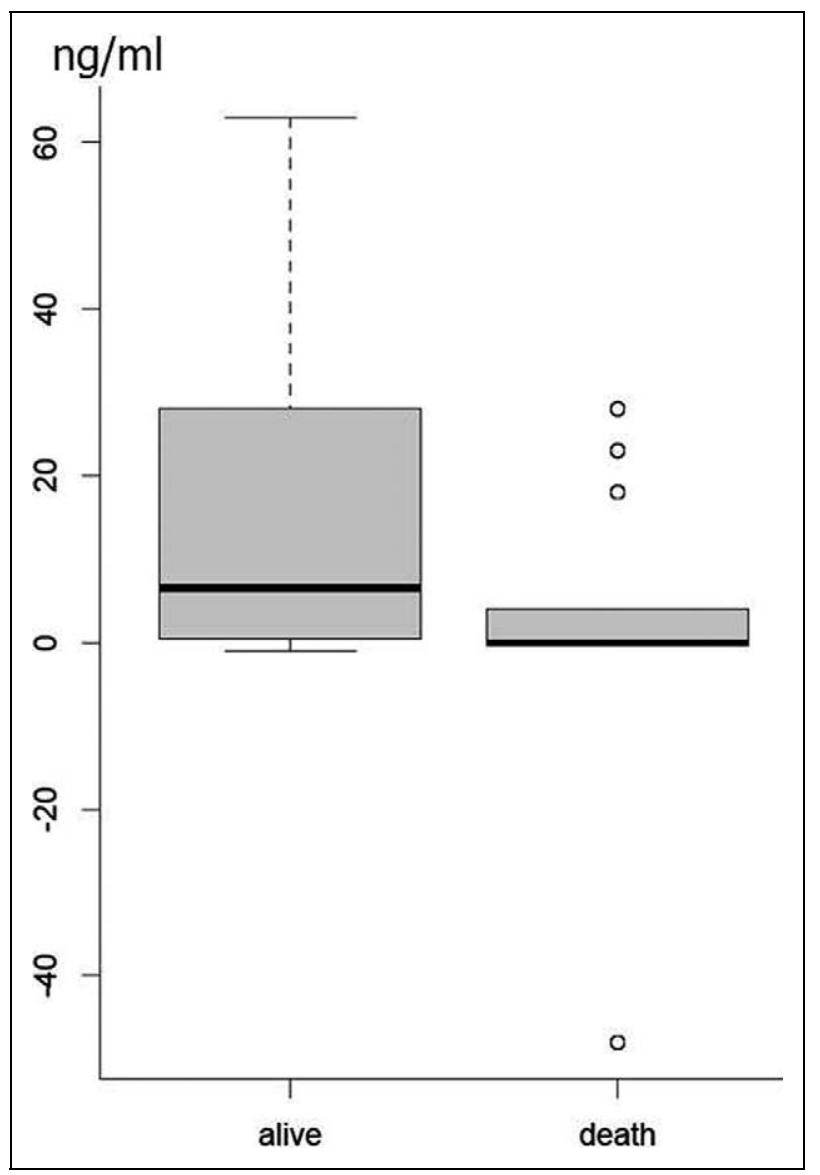

Figure 3. Difference of troponin I levels between first and second postoperative days after extracorporeal membrane oxygenation (ECMO) implantation compared between survivors and nonsurvivors. Decline in levels was significantly $(P=.028)$ higher in survivors.

why patients are prone to low cardiac output syndrome with the need for mechanical support.

\section{Markers for ECMO Survival}

The major goal in this study was to analyze which routine laboratory parameter could serve as a potential marker for ECMO survival. The probability to predict a bridge to recovery or failure thereof, to transplant or to destination therapy often remains unclear, especially in the beginning of extracorporeal life support.

Luyt and colleagues ${ }^{19}$ determined concentrations of TnI, Nterminal fragment of the B-type natriuretic peptide, midregional fragment of the proatrial natriuretic peptide, proadrenomedullin, and copeptin on days 1,3 , and 7 post-ECMO. Neither the absolute values of those biomarkers at days 1,3 , or 7 nor their kinetics during the first week differed between patients with successful of unsuccessful weaning. Sodian and colleagues ${ }^{20}$ analyzed 21 patients with nonischemic cardiomyopathy who received long-term left ventricular assist device (Berlin Heart, Berlin, Germany) support. The plasma B-type natriuretic peptide concentrations decreased significantly after initiation of

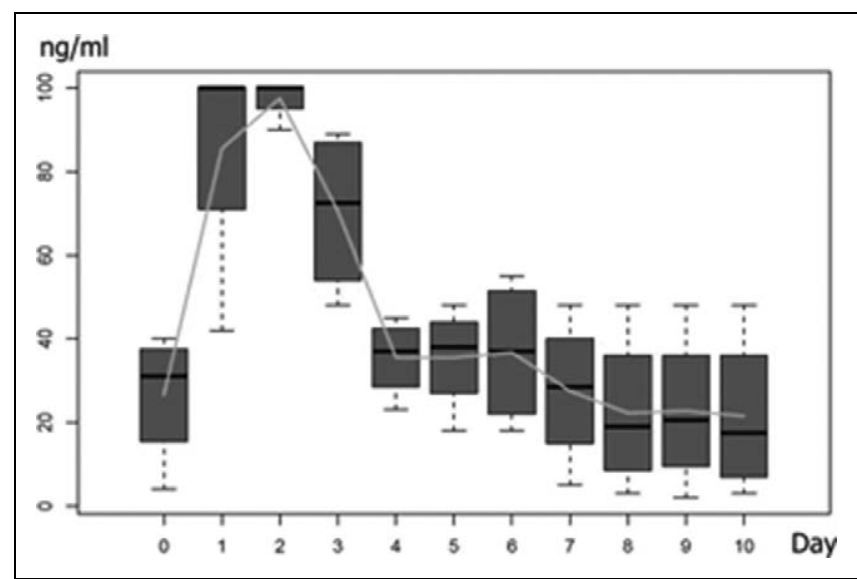

Figure 4. Box plots of median troponin I levels in patients with peak levels at the second postoperative day.

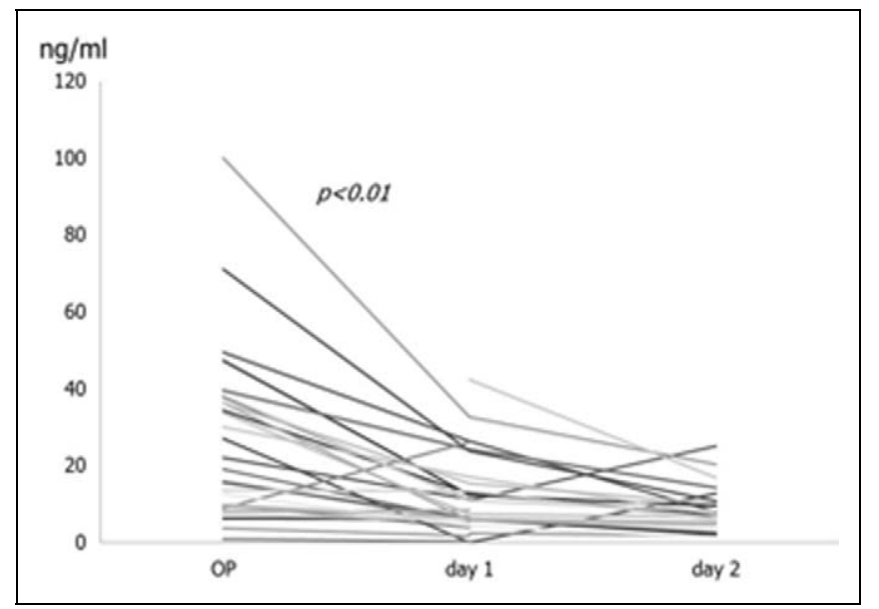

Figure 5. Levels of troponin I (Tnl) from operation to second postoperative day in a control group of hospital survivors younger than one year without extracorporeal membrane oxygenation (ECMO): difference between arrival on ICU and first postoperative day was statistically significant.

circulatory support, and the decline was faster in patients who were subsequently successfully weaned, compared with those who were not. The authors concluded that an early decrease in B-type natriuretic peptide plasma concentration may be indicative of ventricular function recovery during mechanical circulatory support. Whether these results are applicable to patients under ECMO support is a matter of future investigation. Unfortunately, B-type natriuretic peptide was not part of our study protocol. Naruke and colleagues ${ }^{21}$ observed that an abrupt increase in continuously measured end-tidal $\mathrm{CO}_{2}$ concentration, as a sign of increasing pulmonary perfusion and beginning right heart recovery, always preceded successful weaning from ECMO and lacked in patients failing to wean. Their "flexion point" was defined as an initial increase in end-tidal $\mathrm{CO}_{2}$ concentration of $5 \mathrm{~mm} \mathrm{Hg}$ or greater, followed by a continuous rise over the next 12 hours. Lequier and colleagues have demonstrated that the time for serum lactate to normalize on ECMO may be a risk 
factor for a poor mental score at two years' follow-up. ${ }^{22}$ As previously suggested, abnormal $\mathrm{pH}$ and serum lactate values reflect either a global hypoperfusion state before deployment of ECMO or problems in oxygen delivery and extraction after deployment of ECMO. ${ }^{3}$

The TnI is a specific myocardial ischemia marker with a very short half-life (120 minutes) and is cleared primarily by the kidney. Al Badr and colleagues showed no correlation between peak TnI levels and renal function as measured by creatinine clearance in the entire cohort with acute coronary syndrome, and in the subgroups with ST-segment elevation myocardial infarction and non-ST-segment elevation myocardial infarction. The authors concluded that elevated $\mathrm{TnI}$ in the appropriate clinical context in patients with renal insufficiency should be considered as indicative of myocardial ischemia unless proven otherwise. ${ }^{23}$

In our study, we showed a certain correlation between the course of TnI levels and outcomes in patients requiring postcardiotomy ECMO. The refinement of intensive care methods leading to "keeping patients alive" for a certain period has led to a gap between patients successfully weaned and those discharged from the hospital; therefore, we decided to differentiate between "real" survivors and nonsurvivors in our study. In nonsurvivors, levels formed a plateau between the first and the second day, and remained elevated beyond one-week postECMO implantation. All patients in whom peak TnI levels persisted for 48 hours did not survive. As illustrated by our control group with a noneventful perioperative course, $\mathrm{TnI}$ reaches its peak on the operative day, indicating operative trauma, and shows a significant decrease until the next day. Accordingly, early decreasing $\mathrm{TnI}$ levels after complex surgery with postcardiotomy ECMO seem to indicate myocardial recovery with effective hemodynamic support and ventricular unloading. On the other hand, failure of TnI to decrease could be related either to inadequate ECMO support or to ongoing ischemia resulting from major myocardial injury sustained at surgery or a persistent problem with coronary blood flow to the myocardium.

\section{Technical Issues}

In the presence of persisting elevated TnI levels, all technical issues regarding suboptimal ECMO support which could interfere with myocardial recovery should be checked and excluded: first, the role of left atrial venting by a second venous cannula needs to be addressed. We have recently changed our ECMO strategy leading to left ventricular preload reduction and improved biventricular unloading by utilizing biatrial cannulation in patients with an intact atrial septum. Second, high ECMO flows may create an elevated afterload, which may compromise left ventricular decompression. Third, in the presence of left ventricular output, insufficient ventilation may lead to cyanotic coronary perfusion despite optimal arterial saturation achieved by the ECMO oxygenator.

\section{Decision Making}

In general, cardiac catheterization or computed tomography scan should be considered in patients who cannot be weaned from bypass. If residual hemodynamic lesions or insufficient coronary flow are diagnosed, surgical revision is indicated. We feel, however, that the benefit of invasive diagnostics must be weighed against the risks of transport, patient manipulation, exposure to contrast agents, and possible coronary injury. Moreover, there is theoretically a possibility that an immediate revision may result in further cardiac trauma, rather than waiting for the relief of myocardial stunning and cardiac swelling, which is expected by ECMO unloading. The difficult decisionmaking relies on experience and the surgeon's knowledge of intraoperative events and assessment of the likelihood of finding an important remediable problem. The decision must be reached early, before irreversible myocardial injury has occurred. We suggest that the course of TnI levels may assist in this algorithm.

\section{Study Limitations}

A risk-adjusted analysis including all potential variables being associated with ECMO mortality was not part of this study. The methodology chosen does not establish predictive values of troponin levels at 48 hours or of its relationship to levels during the preceding 24 hours. Limitations of the study are inherent to the retrospective nature of data retrieval. Historical influences on outcome related to improvements in ECMO technology, intensive care protocols, and indications for the use of ECMO over the relatively long-study span cannot be excluded. It is difficult to know whether survival on ECMO in those patients with plateau TnI levels at 48 hours could have been improved or influenced by additional diagnostic or interventional cardiac catheterization or by surgical fine-tuning aimed at optimizing hardware issues pertaining to ECMO flows and leading to enhanced ventricular unloading, although the latter were systematically addressed to the best of our knowledge and according to our protocols. Retrospectively, the additional procedures among our six patients with an unsuccessful outcome were performed relatively late.

\section{Conclusions}

Aortic arch hypoplasia was the major congenital anomaly among pediatric patients requiring postcardiotomy ECMO support in our cohort. It is important to consider the trend and time course of change in TnI levels rather than relying entirely on absolute values. Decreasing levels of TnI during the first 48 hours after ECMO deployment seem to be indicative of beginning myocardial recovery. In contrast, plateau TnI levels at 48 hours seem to correlate with a higher likelihood of an unfavorable outcome. Patients should undergo diagnostic and/or surgical revision aiming at optimizing myocardial performance, before assuming irreversible myocardial damage has occurred. 


\section{Declaration of Conflicting Interests}

The author(s) declared no potential conflicts of interest with respect to the research, authorship, and/or publication of this article.

\section{Funding}

The author(s) received no financial support for the research, authorship, and/or publication of this article.

\section{References}

1. Baffes TG, Fridman JL, Bicoff JP, Whitehill JL. Extracorporeal circulation for support of palliative cardiac surgery in infants. Ann Thorac Surg. 1970;10(4): 354-363.

2. Hoskote A, Bohn D, Gruenwald C, Edgell D, Cai S, Adatia I, Van Arsdell G. Extracorporeal life support after staged palliation of a functional single ventricle: subsequent morbidity and survival. J Thorac Cardiovasc Surg. 2006;131(5): 1114-1121.

3. Polimenakos AC, Wojtyla P, Smith PJ, Rizzo V, Nater M, El Zein CF, Ilbawi MN. Post-cardiotomy extracorporeal cardiopulmonary resuscitation in neonates with complex single ventricle: analysis of outcomes. Eur J Cardiothorac Surg. 2011;40(6): 1396-1405; discussion 1405.

4. Kumar TK, Zurakowski D, Dalton H, et al. Extracorporeal membrane oxygenation in postcardiotomy patients: factors influencing outcome. J Thorac Cardiovasc Surg. 2010;140(2): 330-336 e332.

5. Alsoufi B, Al-Radi OO, Nazer RI, et al. Survival outcomes after rescue extracorporeal cardiopulmonary resuscitation in pediatric patients with refractory cardiac arrest. J Thorac Cardiovasc Surg. 2007;134(4): 952-959 e952.

6. Kolovos NS, Bratton SL, Moler FW, et al. Outcome of pediatric patients treated with extracorporeal life support after cardiac surgery. Ann Thorac Surg. 2003;76(5): 1435-1441; discussion 14411432.

7. Thourani VH, Kirshbom PM, Kanter KR, et al. Venoarterial extracorporeal membrane oxygenation (VA-ECMO) in pediatric cardiac support. Ann Thorac Surg. 2006;82(1): 138-144; discussion 144-135.

8. Etievent JP, Chocron S, Toubin G, et al. Use of cardiac troponin I as a marker of perioperative myocardial ischemia. Ann Thorac Surg. 1995;59(5): 1192-1194.

9. Adams JE III, Sicard GA, Allen BT, et al. Diagnosis of perioperative myocardial infarction with measurement of cardiac troponin I. N Engl J Med. 1994;330(10): 670-674.

10. Kociol RD, Pang PS, Gheorghiade M, Fonarow GC, O'Connor $\mathrm{CM}$, Felker GM. Troponin elevation in heart failure prevalence, mechanisms, and clinical implications. $\mathrm{J}$ Am Coll Cardiol. 2010;56(14): 1071-1078.
11. Carrier M, Pellerin M, Perrault LP, Solymoss BC, Pelletier LC. Troponin levels in patients with myocardial infarction after coronary artery bypass grafting. Ann Thorac Surg. 2000;69(2): 435-440.

12. Eigel $P$, van Ingen $G$, Wagenpfeil S. Predictive value of perioperative cardiac troponin I for adverse outcome in coronary artery bypass surgery. Eur J Cardiothorac Surg. 2001;20(3): 544-549.

13. Hirsch R, Dent CL, Wood MK, et al. Patterns and potential value of cardiac troponin I elevations after pediatric cardiac operations. Ann Thorac Surg. 1998;65(5): 1394-1399.

14. Immer FF, Stocker FP, Seiler AM, Pfammatter JP, Printzen G, Carrel TP. Comparison of troponin-I and troponin-T after pediatric cardiovascular operation. Ann Thorac Surg. 1998;66(6): 2073-2077.

15. Haines NM, Rycus PT, Zwischenberger JB, Bartlett RH, Undar A. Extracorporeal life support registry report 2008: neonatal and pediatric cardiac cases. ASAIO J. 2009;55(1): 111-116.

16. Pizarro C, Davis DA, Healy RM, Kerins PJ, Norwood WI. Is there a role for extracorporeal life support after stage I Norwood? Eur J Cardiothorac Surg. 2001;19(3): 294-301.

17. Chow G, Koirala B, Armstrong D, et al. Predictors of mortality and neurological morbidity in children undergoing extracorporeal life support for cardiac disease. Eur J Cardiothorac Surg. 2004; 26(1): 38-43.

18. Massetti M, Tasle M, Le Page O, et al. Back from irreversibility: extracorporeal life support for prolonged cardiac arrest. Ann Thorac Surg. 2005;79(1): 178-183.

19. Luyt CE, Landivier A, Leprince P, et al. Usefulness of cardiac biomarkers to predict cardiac recovery in patients on extracorporeal membrane oxygenation support for refractory cardiogenic shock. J Crit Care. 2012;27(5): 524.e7-14.

20. Sodian R, Loebe M, Schmitt C, et al. Decreased plasma concentration of brain natriuretic peptide as a potential indicator of cardiac recovery in patients supported by mechanical circulatory assist systems. J Am Coll Cardiol. 2001;38(7): 1942-1949.

21. Naruke T, Inomata T, Imai H, et al. End-tidal carbon dioxide concentration can estimate the appropriate timing for weaning off from extracorporeal membrane oxygenation for refractory circulatory failure. Int Heart J. 2010;51(2): 116-120.

22. Lequier L, Joffe AR, Robertson CM, et al. Two-year survival, mental, and motor outcomes after cardiac extracorporeal life support at less than five years of age. J Thorac Cardiovasc Surg. 2008;136(4): 976-983.e973.

23. Al Badr W, Mukherjee D, Kline-Rogers E, et al. Clinical association between renal insufficiency and positive troponin I in patients with acute coronary syndrome. Cardiology. 2004;102(4): 215-219. 